\title{
Magnetic Small Angle X-Ray Scattering
}

\author{
P. Fischer, R. Zeller, G. Schütz, G. Goerigk* and H.-G. Haubold* \\ Universität Augsburg, Institut f. Experim. Phys. II, Memmingerstrasse 6, 86135 Augsburg, Germany \\ * Forschungszentrum Jülich, IFF, 52428 Jülich, Germany
}

\begin{abstract}
We present first experimental results of the extension of X-ray Magnetic Circular Dichroism (X-MCD) to Small Angle X-ray Scattering (SAXS). The purpose of this study is to determine size distributions and correlations lengths in the nanometer range of magnetic precipitates in granular systems. Magnetic scattering curves obtained with a GdFe system at the $\mathrm{Gd} \mathrm{L}_{3}$ edge are compared with reference measurements taken with Anomalous Small Angle $\mathrm{X}$-ray Scattering (ASAXS) at the Fe K-edge. The profile of the scattering curves indicate a correlation maximum. The magnetic scattering intensity can be explained within the frame of X-MCD results and magnetic contributions to the anomalous scattering factors $f^{\prime}$ and $f^{\prime \prime}$.
\end{abstract}

\section{INTRODUCTION}

X-ray Magnetic Circular Dichroism (X-MCD), i.e. the spin-dependent absorption of circular polarized photons [1], has become a powerful tool for the element-specific and symmetry-selective investigation of the local magnetic structure on an atomic scale. Subsequently magnetic effects up to $30 \%$ have been manisfested at spin-orbit splitted L-edges in 3d-, 4f- and 5d-systems. Applying magneto-optical sum-rules [2] it is possible to determine in certain cases spin and orbital moments separately. Even in the EXAFS range a spin-dependent contribution has been manifested [3] and can be used to study the magnetic short-range order even in multi-component systems. The so called multi-path enhancement effect provides magnetic information from a larger spatial dimension up to $20 \AA$ than with conventional EXAFS, as the spin polarization is piled up in triplett/singlett scattering process.

The potential of X-MCD, however, is not restricted to the study of absorption profiles. In principle every method used in spectroscopy or crystallography, where the absorption coefficient is involved, can be extended to its magnetic counterpart.

Recently, nanostructured systems have attracted considerable interest. For instance the occurence of Giant Magnetoresistance (GMR), oscillatory exchange effects and the perpendicular magnetic anisotropy are discussed within fundamental physics of magnetism. But also for technical applications, as e.g. for the development of modern magnetic media granular RE-TM systems are candidates for magneto-optical storage media. Therefore a fundamental understanding of magnetic properties on a $\mathrm{nm}$ or even larger scale is urgently needed.

One common way to study size distributions and correlations of particles in the nm range is the Small Angle X-ray Scattering (SAXS). Using tunable X-rays with high brilliance being available at synchrotron sources the technique of contrast enhancement (Anomalous SAXS=ASAXS) can be engaged, i.e. involving the element-specific anomalous scattering amplitude $f^{\prime}(E)$, which varies with energy by about $20 \%$ in the vicinity of absorption edges, thus providing more detailed structural information.

In a two phase model the scattering contrast $\Delta c$ in SAXS is given by $\left(n_{1} \cdot f_{1}-n_{2} \cdot f_{2}\right)$ with $n_{i}$, $f_{i}$ being the density and atomic scattering amplitudes of phase $i$. The cross section $\frac{d \sigma}{d \Omega}$ is proportional to $\Delta c^{2}$ which in the case of anomalous scattering can be related to $\left(n_{1}=n_{2}\right)$

$$
\frac{d \sigma}{d \Omega} \simeq|f(E, Z)|^{2}=\left|f_{0}+f^{\prime}(E)+i f^{\prime \prime}(E)\right|^{2}
$$

with the atomic form factor $f_{0}=Z$ (atomic number) and $f^{\prime}$ and $f^{\prime \prime}$ being the additional anomalous contributions. $f^{\prime}$ and $f^{\prime \prime}$ are connected to each other via Kramers-Kronig relations, whence $f^{\prime \prime}$ can be related to the absorption coefficient $\mu(E)$ via the optical theorem

$$
f^{\prime \prime}(E)=\frac{m c}{4 \pi e^{2}} \frac{E}{h} \mu(E) .
$$

The basic idea of magnetic anomalous small angle X-ray scattering (MASAXS) is now to achieve the contrast enhancement via the effect of X-MCD in the vicinity of an absorption edge. As the dichroic signal depends on the magnetization of the absorbing atoms the magnetic scattering curves reflect the size distribution and correlation lengths of the magnetic scattering precipitates. 


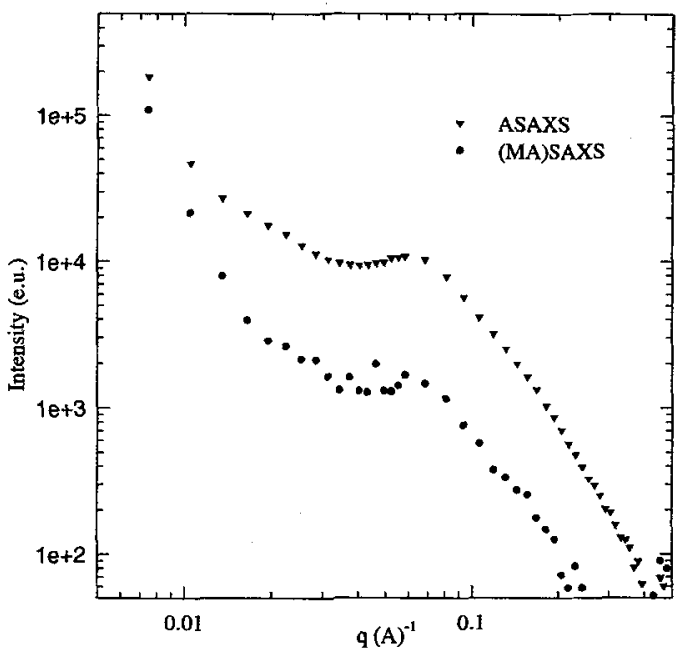

Figure 1: ASAXS at the Fe K-edge in $\mathrm{Gd}_{25} \mathrm{Fe}_{75}$ in comparison with the results of MASAXS at the Gd $\mathrm{L}_{3}$ edge.

Given the magnetic absorption coefficient for parallel/antiparallel alignment of magnetic electrons relative to the photon propagation direction by $\mu^{ \pm}(E)=\mu_{0}(E) \pm \mu_{c}(E)$ the corresponding scattering amplitudes $f^{\prime \pm}(E)$ and $f^{\prime \prime \pm}(E)$ both acquire magnetic contributions $f_{c}^{\prime}(E)$ and $f_{c}^{\prime \prime}(E)$, namely

$$
\begin{aligned}
f^{\prime \pm}(E) & =f^{\prime}(E) \pm f_{c}^{\prime} \\
f^{\prime \prime \pm}(E) & =f^{\prime \prime}(E) \pm f_{c}^{\prime \prime}
\end{aligned}
$$

Inserting eqs.(3,4) into eq.(1) the magnetic cross section, i.e. the difference in scattered intensities for parallel and antiparallel orientation is given by

$$
\left(\frac{d \sigma}{d \Omega}\right)^{+}-\left(\frac{d \sigma}{d \Omega}\right)^{-} \simeq 4\left(f_{0}+f^{\prime}\right) f_{c}^{\prime}+4 f^{\prime \prime} f_{c}^{\prime \prime}
$$

\section{EXPERIMENTAL DETAILS}

We report on the first results obtained at the JUSIFA beamline at HASYLAB/ Hamburg (FRG) with a binary Gd Fe $_{25}$ system. The amorphous film was prepared by coevaporation from two electron-gun sources in a high vacuum system onto a $15 \mu m$ Mylar $^{R}$ foil. For chemical protection the Gd-Fe films of thickness $h=105-115 n m$ were covered with $20-22 \mathrm{~nm}$ Al layers on both sides of the Gd-Fe layer. A stack of 12 foils was enough to achieve a transmission of 0.5 .

The magnetic anisotropy out-of-plane was verified by VSM measurements and Faraday effect results exhibiting a rectangular hysteresis loop with a magnetic coercitive field of $\approx 20 \mathrm{mT}$.

The set-up for the ASAXS measurements at the JUSIFA beamline, described in detail in [4], was modified slightly for the magnetic measurements. In order to illuminate the sample with circular polarized X-rays the inclined view method was used. The beam position control monitor was set to an asymmetric position $2.5 \mathrm{~mm}$ above/below the orbital plane with a slit height of $1.4 \mathrm{~mm}$. The beam height position was checked by recording the beam profile after each scan. The calculated degree of circular polarization amounts to $\approx 70 \%$. A small solenoid provided magnetic flux densities up to $60 \mathrm{mT}$ with its field direction pointing parallel/antiparallel to the photon beam direction sufficient to saturate the sample.

The magnetic contrast scattering curve was then obtained according to eq.(5) by recording two scattering profiles with parallel/antiparallel orientation of the magnetization relative to the photon propagation direction at an incident photon energy where the maximum dichroic effect for $\mathrm{Gd}$ is expected.

\section{RESULTS AND DISCUSSION}

In fig. 1 the magnetic scattering curve intensity obtained in that way vs. momentum transfer $q$ measured at the Gd $\mathrm{L}_{3}$ edge $(E=7243 \mathrm{eV})$ is presented in comparison with the ASAXS profile obtained at the corresponding Fe-K edge. The similarity of both profiles is obvious. The pronounced maximum at $q \sim 0.06 \AA^{-1}$ can be interpreted as a correlation maximum with $l_{c} \sim 100 \AA$ of the Gd precipitates.

This feature occurs also in the magnetic profile. The structural parameters could be determined by fitting a log-normal distribution to the experimental data. 


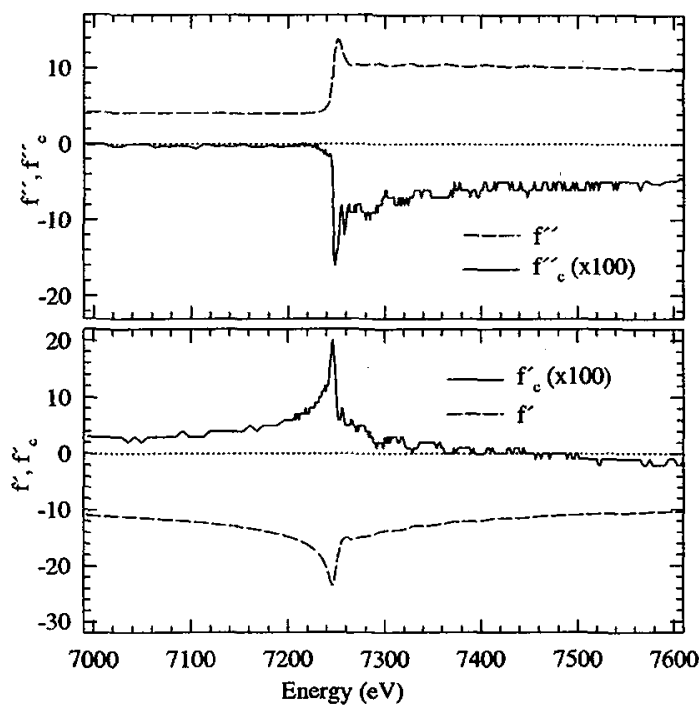

Figure 2: Anomalous scattering factors $f^{\prime}, f_{c}^{\prime}$ deduced from magnetic EXAFS measurements at the Gd $L_{3}$ edge (top panel) and $f^{\prime \prime}, f_{c}^{\prime \prime}$ calculated via Kramers-Kronig relations (bottom panel). The magnetic values are magnified by a factor 100 .

The intensity of the MASAXS profile can be related to accurate experimental measurements of magnetic EXAFS in a wide range around the $\mathrm{Gd} \mathrm{L}_{3}$ edge with the help of the optical theorem and Kramers-Kronig relation. The obtained results for $f^{\prime}, f_{c}^{\prime}$ and $f^{\prime \prime}, f_{c}^{\prime \prime}$, resp., as function of energy are shown in fig. 2. The relevant values at $E=7243 e V$ for $f_{c}^{\prime}$ amount to 0.2 , while $f_{c}^{\prime \prime} \approx-0.16$. According to eq. (5) the magnetic contrast reaches a value of 32.9 . For the conventional ASAXS measurement the contrast enhancement taken at $E=7109 \mathrm{eV}$ and $E=6843 \mathrm{eV}$ amounts to -242.7. Therefore the contrast ratio between ASAXS and MASAXS can be directly seen in the ratio of scattered intensities in fig. 1. This ratio is larger than the dichroic effect at $E=7243 \mathrm{eV}$ observed in absorption due to the large contribution of $f_{c}^{\prime}$.

It has to be mentioned that a similar behaviour has already been observed in magnetic resonant scattering in $\mathrm{Ni}$ [5], where the flipping ratio $\left(I^{+}-I^{-}\right) /\left(I^{+}+I^{-}\right)$at $\sim-10 \mathrm{eV}$ before the Ni K-edge exhibits a constant ratio of $\approx 7 \%$, a feature, which could not be observed with linear polarized light [6].

Although the magnetic profile in fig.1 follows roughly the non-magnetic scattering curve, slight deviations can be observed for small $q$-values, i.e. for large particle sizes. This can be attributed to an enhanced magnetization in the formation of magnetic domains with a corresponding thickness of $\sim 100 \mathrm{~nm}$, which could be observed recently in magnetic X-ray microscopy [7].

\section{OUTLOOK AND CONCLUSION}

We have presented the first results of Magnetic Small Angle X-ray Scattering which allows new insights into structural characteristics of magnetic particles in granular systems and diluted alloys, relevant both in the frame of Giant Magnetoresistance and perpendicular anisotropy and in the frame of technological applications. Therefore a detailed analysis of the scattering profiles obtained with this new method can reveal new and important both electronic and magnetic structural information necessary for a better understanding of magnetism on a $n m$ scale.

\section{Acknowledgments}

We like to thank D. Raasch from Philips/Aachen,FRG who prepared the sample for us. This work was supported by the German federal ministery of education, science, research and technology (BMBF) project No. 05621 WAA.

\section{References}

[1] G. Schütz et al., Phys. Rev. Lett 58 (1987) 737

[2] B.T. Thole et al., Phys. Rev. Lett. 68(12) (1992) 1943 P. Carra et al., Phys. Rev. Lett. 70(5) (1993) 694

[3] see e.g G. Schütz, Plenary talk at XAFS9 (1996)

[4] H.-G. Haubold et al., Rev. Sci. Instr. 60(7) (1989) 1943

[5] J. Hunecke et al., HASYLAB Annual report (1990)

[6] K. Namikawa et al., J.Phys.Soc.Japan 54,11 (1985) 4099

[7] P. Fischer et al., subm. to Z Phys. B (1996) 